\title{
Diversity of Fern Species (Pteridophyta) in the Karst Sangkulirang - Mangkalihat
}

\author{
Febrian Jeka Risky ${ }^{1}$, Matius Paulus ${ }^{1}$, Sutedjo $^{1}$ Rita Diana $^{1, *}$ \\ ${ }^{1}$ Faculty of Forestry, Mulawarman University, Kampus Gn. Kelua, Jl. Ki Hajar Dewantara, Samarinda, Indonesia \\ *Corresponding author. Email: ritadiana@fahutan.unmul.ac.id
}

\begin{abstract}
This study aims to determine the diversity of fern vegetation in the Sangkulirang- Mangkalihat karst area of Berau and East Kutai Regency. The research was carried out for two months in six locations. The plotting method used the purposive sampling method, with a plot size of $10 \mathrm{~m}$ x $10 \mathrm{~m}$ as many as 15 at each location. The research location of the karst area of Sangkulirang - Mangkalihat was found 4,871 individuals from 55 species, 39 genera, and 23 families. The most abundant species is Selaginella willdenowii (Desv. ex Poir.) Baker. Diversity Index (H') was ranging from 1.37 to 2.36. Dominance Index (C) in all research locations closed to 0 , so it can be said that there were no dominant species in each location. Evenness Index (e) in Biatan Ulu was more than 0.96, while other locations were more than 0.51. Similarity Index (ISs) in all study locations was less than $50 \%$, so it means that all species in each location are different.
\end{abstract}

Keywords: Karst, Fern, Selaginella willdenowii

\section{INTRODUCTION}

Karst forests that grow on rocks are limestone containing calcium carbonate or calcite which is easily dissolved by rainwater, which led to the formation of cracks and tunnels that resembled relief forming morphology. Typical causes of karst forests are that the appearance became more specific than other forests and the exchange of cations that are higher than the soil in the lowland forests [1]. One species of biodiversity of flora in Indonesia is ferns [2], a homospore plant that can live easily on a wide variety of habitats and anywhere either an epiphyte terrestrial epiphyte, terrestrial as well as in water [3].

\section{METHODS}

The research was carried out for two months in 6 locations. The plotting method is done using the purposive sampling method, with a plot size of $10 \times 10$ as many as 15 at each location.

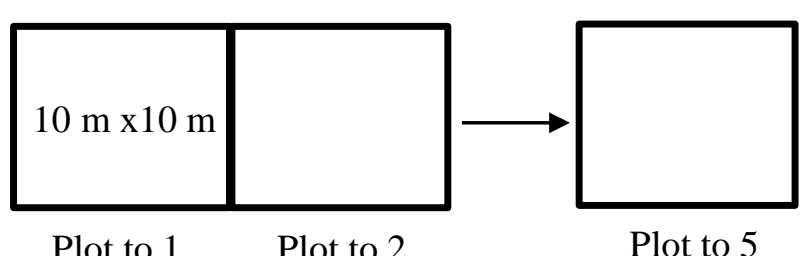

Plot to $1 \quad$ Plot to 2

Plot to 5

Figure 1. The Scheme of Making Plots.

\section{RESULTS AND DISCUSSION}

\subsection{Species Diversity and Density}

The individual number of ferns found in the whole plot location in the Sangkulirang karst area Mangkalihat was 4,871 individuals from 55 species, 39 genera, and 23 families. The most abundant species was Selaginella willdenowii (Desv. ex Poir.) Baker. Furthermore, the number of individual fern species found is indicated in Table 1.

\subsection{Diversity ( $\left.H^{\prime}\right)$, Dominance $(C)$, and Equity (e) Index}

The result of the calculation of the diversity index $\left(\mathrm{H}^{\prime}\right)$, dominance index (C), and equity index (e) 
Table 1. Ferns in Sangkulirang Mangkalihat - Karst Area

\begin{tabular}{lrrr}
\hline Location & Individual & Species & Family \\
\hline Suaran & 200 & 15 & 8 \\
Tondoyan & 2405 & 32 & 18 \\
Gunung Gergaji & 1355 & 20 & 14 \\
Biatan Ulu & 358 & 12 & 7 \\
Lobang Kelatak & 448 & 19 & 11 \\
Biduk-Biduk - Teluk Sulaiman & 113 & 9 & 6 \\
\hline
\end{tabular}

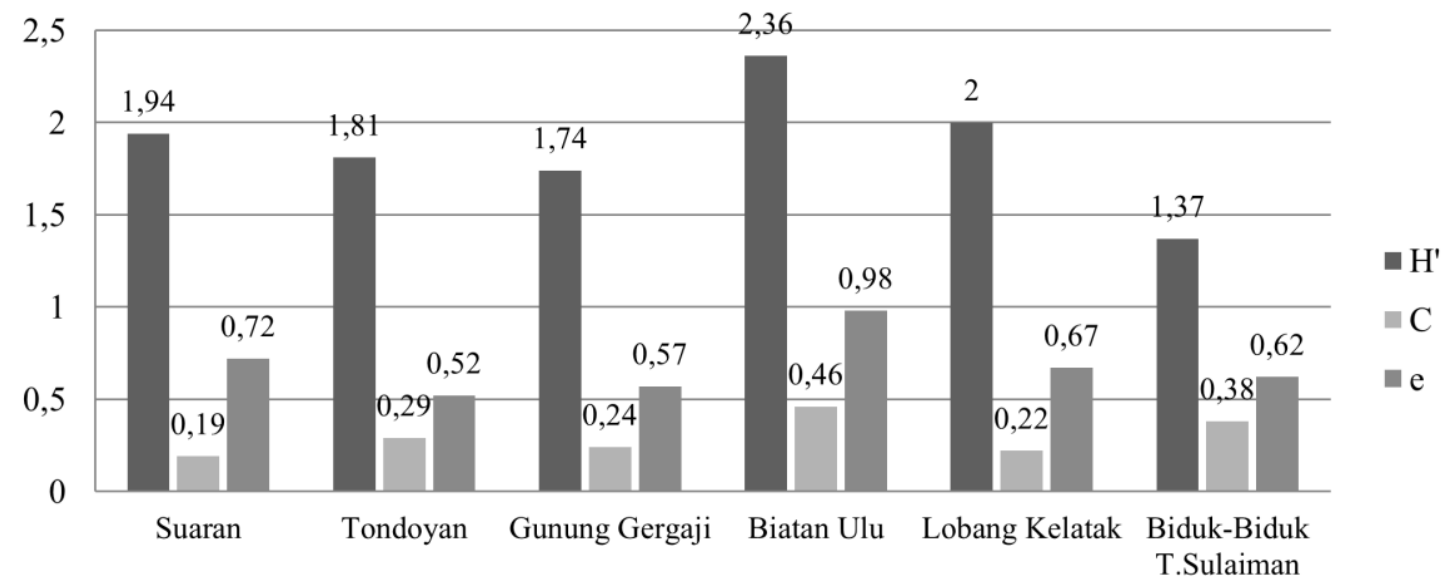

Figure 2 The diversity index ( $\left.\mathrm{H}^{\prime}\right)$, dominance index $(\mathrm{C})$ and equity index (e)

Limestone Mountain Forest area in the Sangkulirang Mangkalihat can be seen in Figure 2.

From Figure 2, the value diversity $\left(\mathrm{H}^{\prime}\right)$ in Suaran was 1.94, Tondoyan was 1.81 , Gunung Gergaji was 1.74, Biatan Ulu was 2.36, Lobang Kelatak was 2.00, and Biduk-Biduk - Teluk Sulaiman was 1.37. Index Dominance (C) in Suaran was 0.19, Tondoyan was 0.29, Gunung Gergaji was 0.24, Biatan Ulu was 0.46, Lobang Kelatak was 0.22, and Biduk-Biduk - Teluk Sulaiman was 0.38. Value Equity (e) of Suaran was 0.72, Tondoyan was 0.52, Gunung Gergaji was 0.57, Biatan Ulu was 0.98, Lobang Kelatak was 0.67, and BidukBiduk - Teluk-Sulaiman was 0.63 .

\subsection{Similarity index (ISs)}

The Similarity Index (ISs) of Limestone Mountain Forest Sangkulirang - Mangkalihat can be seen in Table 2. From Table 3, Index Similarity (ISs) between Suaran and Tondoyan was $32.33 \%$, Lobang Kelatak and BidukBiduk - Teluk Sulaiman was $9.6 \%$. The similarities on all location research were not equal, between 9.6$32.33 \%$.

\section{CONCLUSIONS}

a) The individual number of ferns in the karst area Sangkulirang - Mangkalihat was 4871 individuals from 55 species, 39 genera, and 23 families. The most abundant species was Selaginella willdenowii (Desv. ex Poir.) Baker.

b) The value of diversity index (H') of ferns in the karst area of Sangkulirang-Mangkalihat can tell that diversity in all locations did not exist.

c) The value of dominance index (C) ferns karst area in the Sangkulirang - Mangkalihat on all research locations did not reach one, so it can be said that there are no dominant species in each location.

d) Index value equity (e) of ferns in the karst area of Sangkulirang - Mangkalihat was only distributed pertained evenly in Biatan Ulu, showing the species' stability at that location.

e) The value of the index of similarity (ISs) of ferns in the karst area of Sangkulirang - Mangkalihat has an index value of $50 \%$. 
Table 2. The result of calculation of Similarity Index (ISs)

\begin{tabular}{|c|c|c|c|c|c|c|}
\hline Location & & & & & & \\
\hline Suaran & - & & & & & \\
\hline Tondoyan & 32.33 & - & & & & \\
\hline $\begin{array}{l}\text { Gunung } \\
\text { Gergaji }\end{array}$ & 21.67 & 21.77 & - & & & \\
\hline Biatan Ulu & 11.93 & 11.58 & 11.48 & - & & \\
\hline $\begin{array}{l}\text { Lobang } \\
\text { Kelatak }\end{array}$ & 20.80 & 20.65 & 20.76 & 21.27 & - & \\
\hline $\begin{array}{l}\text { Biduk2 - T. } \\
\text { Sulaiaman }\end{array}$ & 9,53 & 9.32 & 9.38 & 10.09 & 9.6 & - \\
\hline Iss & Suaran & Tondoyan & $\begin{array}{l}\text { Gunung } \\
\text { Gergaji }\end{array}$ & Biatan Ulu & $\begin{array}{l}\text { Lobang } \\
\text { Kelatak }\end{array}$ & $\begin{array}{c}\text { Biduk2 - T } \\
\text { Sulaiman }\end{array}$ \\
\hline
\end{tabular}

\section{REFERENCES}

[1] A. Achmad, Rahasia Ekosistem Hutan Bukit Kapur. Brilian Internasional. Surabaya, 2011. [Indonesian]

[2] S. Sastrapraja, J.J. Afriastini, Kerabat Paku, 103. LBN LIPI, Bogor, 1985. [Indonesian]

[3] Widhiastuti, T.A. Retno, W.D.P. Aththorick, Sari, Struktur dan Komposisi Tumbuhan Paku-Pakuan di Kawasan Hutan Gunung Sinabung Kabupaten Karo, Jurnal Biologi Sumatera, 2006, pp. 38-41. [Indonesian] 


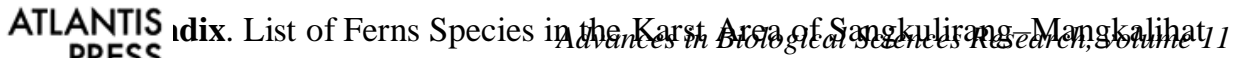
PRESS

\begin{tabular}{|c|c|c|c|}
\hline No & Scientif Name & Family & $\mathrm{N}$ \\
\hline 1 & Adiantum sp. & Pteridaceae & 13 \\
\hline 2 & Antrophyum sp. & Pteridaceae & 3 \\
\hline 3 & Antrophyum callifolium Blume & Pteridaceae & 92 \\
\hline 4 & Antrophyum parvulum Blume & Pteridaceae & 7 \\
\hline 5 & Antrophyum semicostatum Blume & Pteridaceae & 55 \\
\hline 6 & Asplenium batuense Alderw. & Aspleniaceae & 5 \\
\hline 7 & Asplenium nidus $\mathrm{L}$. & Aspleniaceae & 20 \\
\hline 8 & Asplenium robustum Blume & Aspleniaceae & 20 \\
\hline 9 & Asplenium squamulatum Blume & Aspleniaceae & 40 \\
\hline 10 & Bolbitis sp. & Dryopteridaceae & 239 \\
\hline 11 & Bolbitis heteroclita (C. PresI) Ching & Dryopteridaceae & 9 \\
\hline 12 & Cyclopeltis sp. & Lomariopsidaceae & 6 \\
\hline 13 & Cyclopeltis semicordata (Sw.) J. Sm. & Lomariopsidaceae & 209 \\
\hline 14 & Doryopteris ludens (Wall. ex Hook.) J. Sm. & Pteridaceae & 2 \\
\hline 15 & Drynaria sparsisora (Desv.) T. Moore & Polypodiaceae & 160 \\
\hline 16 & Heterogonium pinnatum (Copel.) Holttum & Tectariaceae & 671 \\
\hline 17 & Lepisorus longifolius (Blume) Holttum & Polypodiaceae & 1 \\
\hline 18 & Lygodium sp. & Lygodiaceae & 1 \\
\hline 19 & Lygodium circinnatum (Burm. f.) Sw. & Lygodiaceae & 478 \\
\hline 20 & Lygodium longifolium (Willd.) Sw. & Lygodiaceae & 4 \\
\hline 21 & Mesophlebion motleyanum (Hook.) Holttum & Thelypteridaceae & 3 \\
\hline 22 & Microlepia sp. & Dennstaedtiaceae & 1 \\
\hline 23 & Nephrolepis cordifolia (L.) C. Pres/ & Nephrolepidaceae & 6 \\
\hline 24 & Nephrolepis radicans (Burm.) Kuhn & Nephrolepidaceae & 182 \\
\hline 25 & Neprolephis sp. & Nephrolepidaceae & 1 \\
\hline 26 & Pleocnemia conjugata (Blume) C. PresI & Tectariaceae & 2 \\
\hline 27 & Pronephrium sp. & Thelypteridaceae & 33 \\
\hline 28 & Pronephrium triphyllum (Sw.) Holttum & Thelypteridaceae & 46 \\
\hline 29 & Pteridium sp. & Dennstaedtiaceae & 51 \\
\hline 30 & Pteris $s p$ & Pteridaceae & 11 \\
\hline 31 & Pteris ensiformis Burm. $f$. & Pteridaceae & 1 \\
\hline 32 & Pyrrosia angustata (Sw.) Ching & Polypodiaceae & 1 \\
\hline 33 & Selaginella sp. & Selaginellaceae & 3 \\
\hline 34 & Selaginella intermedia Spring & Selaginellaceae & 22 \\
\hline 35 & Selaginella willdenowii (Desv. ex Poir.) Baker & Selaginellaceae & 1771 \\
\hline 36 & Sp 14 & - & 8 \\
\hline 37 & Sp1 & - & 1 \\
\hline 38 & Sp10 & - & 5 \\
\hline 39 & $S p 11$ & - & 1 \\
\hline 40 & Sp12 & - & 1 \\
\hline 41 & Sp13 & - & 1 \\
\hline 42 & $s p 2$ & - & 2 \\
\hline 43 & $s p 3$ & - & 1 \\
\hline 44 & $s p 4$ & - & 292 \\
\hline 45 & $s p 5$ & - & 2 \\
\hline 46 & sp 6 & - & 26 \\
\hline 47 & $S p 7$ & - & 1 \\
\hline 48 & $S p 8$ & - & 1 \\
\hline 49 & Sp 9 & - & 2 \\
\hline 50 & Sphaerostephanos heterocarpus (Blume) Holttum & Thelypteridaceae & 6 \\
\hline 51 & Tectaria sp. & Tectariaceae & 262 \\
\hline 52 & Tectaria crenata Cav. & Tectariaceae & 2 \\
\hline 53 & Thelypteris curta (Christ) C.F. Reed & Thelypteridaceae & 7 \\
\hline 54 & Thelypteris simulata (Davenp.) Nieuwl. & Thelypteridaceae & 78 \\
\hline 55 & Vittaria $s p$. & Pteridaceae & 11 \\
\hline
\end{tabular}

